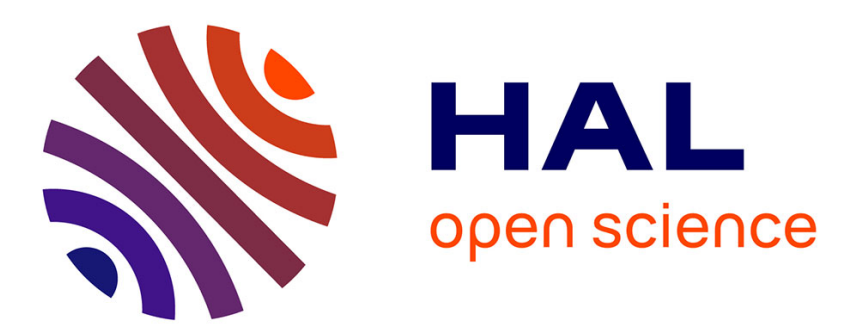

\title{
Utilisation d'une formulation mixte (éléments finis-intégrale de frontière) pour modéliser les systèmes électromagnétiques
}

\author{
F. Bouillault, A. Razek
}

\section{- To cite this version:}

F. Bouillault, A. Razek. Utilisation d'une formulation mixte (éléments finis-intégrale de frontière) pour modéliser les systèmes électromagnétiques. Revue de Physique Appliquée, 1986, 21 (10), pp.595-601. 10.1051/rphysap:019860021010059500 . jpa-00245477

\section{HAL Id: jpa-00245477 https://hal.science/jpa-00245477}

Submitted on 1 Jan 1986

HAL is a multi-disciplinary open access archive for the deposit and dissemination of scientific research documents, whether they are published or not. The documents may come from teaching and research institutions in France or abroad, or from public or private research centers.
L'archive ouverte pluridisciplinaire HAL, est destinée au dépôt et à la diffusion de documents scientifiques de niveau recherche, publiés ou non, émanant des établissements d'enseignement et de recherche français ou étrangers, des laboratoires publics ou privés. 


\title{
Utilisation d'une formulation mixte (éléments finis-intégrale de frontière) pour modéliser les systèmes électromagnétiques
}

\author{
F. Bouillault et A. Razek \\ Laboratoire de Génie Electrique de Paris, E.S.E., Univ. Paris VI et Paris XI, U.A. $\mathrm{n}^{\circ} 127$ au C.N.R.S., \\ Plateau du Moulon, 91190 Gif sur Yvette, France
}

(Reçu le 21 mars 1986, révisé le 2 juin, accepté le 20 juin 1986)

\begin{abstract}
Résumé. - Dans cet article, nous présentons une formulation intégrale de Trefftz appliquée à l'étude des phénomènes électromagnétiques. Nous montrons comment, en couplant cette méthode avec celle des éléments finis, on peut étendre le champ d'investigation de la méthode des éléments finis. Pour valider la méthode, nous modélisons un électro-aimant, les résultats numériques sont comparés aux résultats expérimentaux.
\end{abstract}

Abstract. - In this paper, the study of electromagnetic phenomenon using the Trefftz integral method is presented. We show how to combine this method with finite elements method and how such hybrid formulation can be used to study both linear or infinite domains. An electro-magnet is studied with the help of this hybrid formulation and calculated results are compared with experimental ones to verify the validity of the method.

\section{Nomenclature.}

$A$ potentiel vecteur

$\frac{\partial A}{\partial n}$ dérivée normale du potentiel vecteur

$\nu$ réluctivité

$\delta(x, y)$ fonction de Dirac

$\beta_{j}$ coefficient du point source fictif $j$

$[A]$ vecteur composé des valeurs nodales de $A$

$[\beta]$ vecteur composé des valeurs des points sour-

ces

$d_{i}$ distance du point source $i$ au nœud associé

$\gamma_{i}$ distance du nœud $i$ au nœud $i+1$

$\theta_{0}$ périodicité spatiale des phénomènes.

\section{Introduction.}

Pour le calcul des champs dans les structures électromagnétiques, la méthode des éléments finis présente des avantages par rapport aux méthodes intégrales. Les principaux sont l'obtention de matrices bien conditionnées à structure bande et la possibilité de traiter des phénomènes non linéaires. Cependant la représentation de domaines infinis par la méthode des éléments finis peut devenir fort onéreuse et même impossible. Ce genre de problème est, par contre, très bien adapté à un traitement par une méthode intégrale. Aussi parât-il judicieux, dans certains cas, de coupler les deux méthodes ; au sein d'un même système, certaines régions sont modélisées par des éléments finis alors que les autres le sont par des méthodes intégrales [1, 2]. Il est alors nécessaire d'assurer les conditions de continuité magnétique entre ces régions.

L'utilisation de la méthode intégrale permet de réduire le nombre de nœuds nécessaire pour une modélisation fidèle. Ce gain sur l'espace mémoire peut compenser largement les pertes dues à l'élargissement de la bande des matrices de raideur. L'utilisation des méthodes intégrales peut également être utile dans le traitement de la prise en compte du mouvement. Les techniques utilisées, dans une modélisation par éléments finis, consistent à considérer un « domaine linéaire de liaison » $[3,4]$ entre les milieux en mouvement, ceci contribue à une augmentation de la bande des matrices. L'élément étant linéaire et la bande des matrices étant déjà augmentée dans une formulation par éléments finis, il peut être intéressant d'utiliser une méthode intégrale pour modéliser l'élément tampon.

\section{Méthode intégrale de frontière.}

Généralement, la méthode intégrale classique qui sert à modéliser un système où l'équation à résoudre est :

$$
\Delta \phi=0
$$


est basée sur l'utilisation de l'identité de Green. La solution au point $M\left(x_{0}, y_{0}\right)$ s'écrit :

$$
\phi\left(x_{0}, y_{0}\right)=\int_{c}\left(G \frac{\partial \phi}{\partial n}-\phi \frac{\partial G}{\partial n}\right) \mathrm{d} c
$$

où $G$ est la fonction de Green, solution de l'équation

$$
\Delta G=-\delta\left(x_{0}, y_{0}\right) \text {. }
$$

Pour résoudre l'égalité (2), il est nécessaire de discrétiser $\phi$ et $\frac{\partial \phi}{\partial n}$. L'intégrale intervenant dans peut alors être calculée numériquement en utilisant une intégration de Gauss.

Dans cet article, une alternative à cette méthode est présentée. Il s'agit de la méthode de Trefftz $[6,7]$ qui semble présenter certains avantages. Cette méthode étant peu employée en électromagnétisme, nous allons rappeler son principe et les précautions à prendre pour obtenir des résultats corrects.

\section{Principe de la méthode de Trefitz.}

La méthode de Trefftz consiste à approximer à l'intérieur d'un domaine $\Omega$, une fonction $\phi(x, y)$ sous forme d'une série de produit de fonctions de forme $N_{j}(x, y)$ et de coefficients $\beta_{j}$. Ceci n'est pas sans rappeler la méthode des éléments finis, mais dans le cas de la méthode de Trefftz, les fonctions $N_{j}$ sont choisies de telle façon que l'équation différentielle régissant $\phi$ soit satisfaite dans $\Omega$ a priori. Les coefficients $\beta_{j}$ n'ont pas de signification physique, ils jouent le rôle de sources fictives en des points $j$ et servent à assurer la continuité de la fonction $\phi$ sur le contour $\Gamma$ du domaine $\Omega$. La formulation du macro-élément exposé dans les articles $[5,8]$ apparaît alors comme basée sur l'utilisation de la méthode de Trefftz pour un domaine de forme particulière.

Dans le cas général où il faut déterminer le potentiel vecteur vérifiant l'équation de Laplace, une solution est

$$
A(m)=\sum_{j=1}^{N} N_{j}\left(r_{m j}\right) \beta_{j}
$$

avec

$$
N_{j}\left(r_{m j}\right)=\log \frac{1}{r_{m j}}
$$

$r_{m j}$ présente la distance du point $m$ au point source $j$. Les singularités pour $A$ aux points $j$ sont évitées si ces points $j$ n'appartiennent pas à $\Omega$.

Si l'on traduit l'égalité (4) pour $N$ points appartenant au contour $\Gamma$, nous pouvons écrire matriciellement :

$$
[A]=[P][\beta]
$$

où $[A]$ est le vecteur colonne contenant les $N$ valeurs nodales du potentiel vecteur appartenant au contour $\Gamma$.

$[P]$ est une matrice carrée dont le terme général $P_{i j}$ est $\log \frac{1}{r_{i j}}$ où $r_{i j}$ désigne la distance séparant le nœud $i$ sur le contour $\Gamma$ du point $j$ (Fig. 1).

$[\beta]$ est le vecteur contenant les valeurs des $N$ sources fictives.

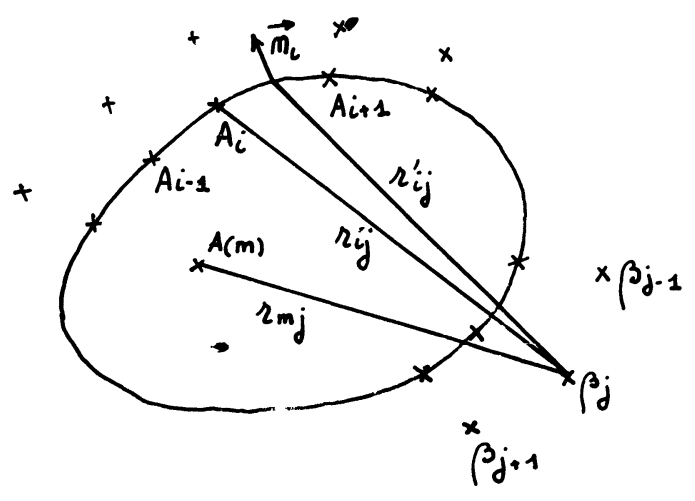

Fig. 1. - Domaine d'étude.

[Studied domain.]

Les relations de continuité magnétique devant être vérifiées sur le contour $\Gamma$ du domaine $\Omega$; il faut assurer la continuité de la composante tangentielle du champ magnétique. Celle-ci peut être calculée à partir de la dérivée normale du potentiel vecteur qui a pour expression :

$$
\frac{\partial A(m)}{\partial n}=\sum_{j=1}^{N}-\frac{\mathbf{r}_{m j} \cdot \mathbf{n}_{m}}{r_{m j}^{2}} \boldsymbol{\beta}_{j}
$$

si l'on calcule la valeur de $\frac{\partial A}{\partial n}$ au milieu de chacun des $N$ segments du contour $\Gamma$, nous avons l'égalité matricielle :

$$
\left[\frac{\partial A}{\partial n}\right]=[Q][\beta]
$$

la matrice $[Q]$ a pour terme général $-\frac{\mathbf{r}_{i j}^{\prime} \cdot \mathbf{n}_{i}}{r_{i j}^{\prime 2}}$.

$r_{i j}^{\prime}$ représente la distance du milieu du segment $i$ au point source $j$ (Fig. 1).

Après élimination du vecteur $[\beta]$ des relations (5) et (7), nous obtenons :

$$
\begin{aligned}
{\left[\frac{\partial A}{\partial n}\right] } & =[Q][P]^{-1}[A] \\
& =[R][A]
\end{aligned}
$$

où $[P]^{-1}$ représente la matrice inverse de $[P]$. Les fonctions $\log \frac{1}{r_{m j}}$ étant indépendantes, la matrice $[P]$ est inversible si les point $j$ sont distincts. 
Comme dans une méthode intégrale de frontière, la relation (8) permet de relier les valeurs nodales du potentiel vecteur $A$ sur le contour $\Gamma$ aux valeurs des dérivées normales de $A$. Les avantages de la méthode résident dans une formulation informatique simple de la relation (8), celle-ci ne nécessite, en particulier, aucune intégration numérique.

3.1 CHOIX DE POINTS SOURCES. - La position des points sources $j$ influe sur la précision des calculs. Ainsi la nécessité de définir « un contour source» apparaît comme un inconvénient de la méthode. Pour voir l'influence de la position de ce contour, nous avons considéré un exemple simple (Fig. 2a) où la solution exacte peut être obtenue analytiquement. Le domaine étudié (Fig. 2a) représente un anneau de rayon extérieur $R_{1}$ et de rayon intérieur $R_{2}$. La discrétisation comporte 80 nœuds dont 40 sont répartis uniformément sur le contour extérieur $\Gamma_{1}$ et 40 sur le contour intérieur $\Gamma_{2}$. Nous avons comparé la valeur de la dérivée normale du potentiel vecteur sur les contours $\Gamma_{1}$ et $\Gamma_{2}$ obtenue analytiquement et numériquement suivant la position des points " sources ». L'examen de l'évolution de l'erreur sur le calcul de la dérivée normale (Fig. 2b) montre que les résultats sont corrects si l'on choisit la distance $d$, entre chaque point source $j$ et le nœud $i$ correspon-

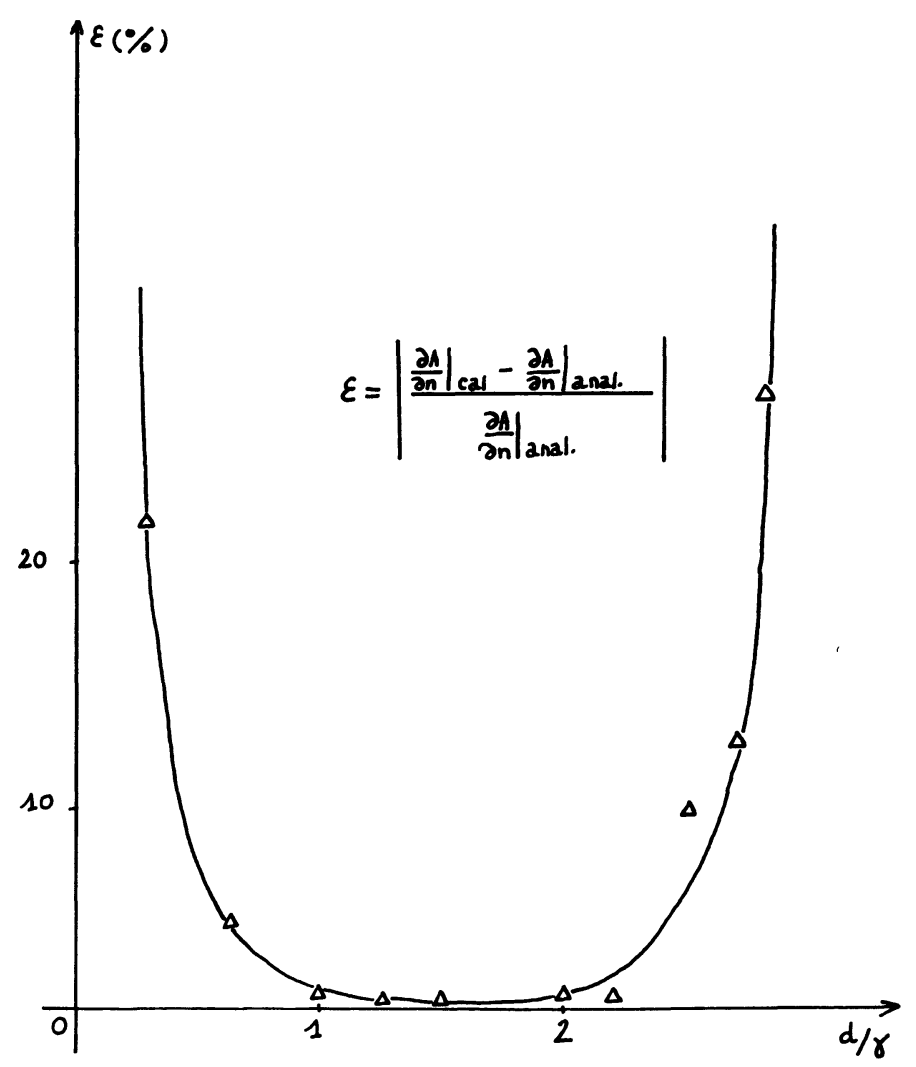

Fig. 2a. - Evolution de l'erreur relative en fonction de la position des points sources.

[Relative error evolution as a function of the source points position.]

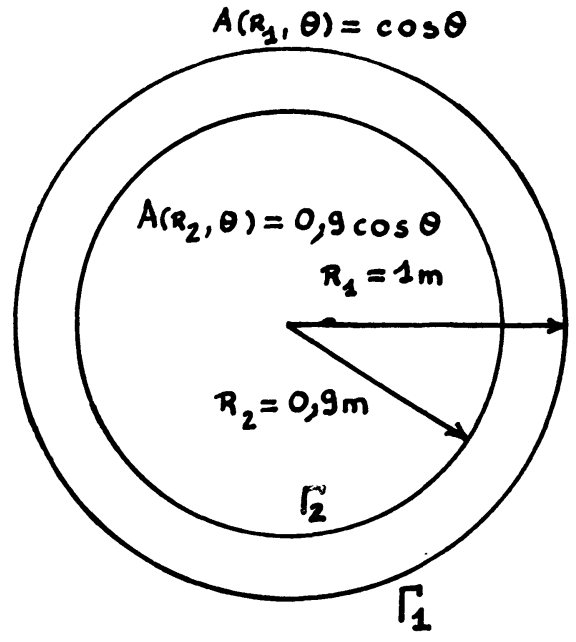

Fig. 2b. - Domaine modélisé en forme d'anneau.

[Domain with a ring form.]

dant sur le contour, comprise entre une à deux fois la distance $\gamma$ entre les nœuds. Dans la présente étude, les points « sources » sont situés sur deux cercles de rayon $R_{1}+d$ et $R_{2}-d$. Dans le cas général où les écarts entre les nœuds du contour sont différents, on peut écrire cette condition comme :

$$
\frac{\gamma_{i}+\gamma_{i-1}}{2}<d_{i}<\gamma_{i}+\gamma_{i-1}
$$

où $d_{i}$ représente la distance entre le nœud $i \mathrm{du}$ contour $\Gamma$ et son point source.

$\gamma_{i}$ représente la distance entre le nœud $i$ et le nœud $i+1$;

$\gamma_{i-1}$ représente la distance entre le nœud $i-1$ et le nœud $i$.

A chaque point du contour $\Gamma$, on associe un point source dont la position dépend du découpage du contour $\Gamma$.

3.2 CONSIDÉRATION DE LA PÉRIODICITÉ. - Dans l'étude numérique de machines électriques, un point important est le traitement des conditions aux limites. Il est rarement nécessaire de considérer toute la machine; dans une étude bidimensionnelle, les conditions de périodicité ou d'anti-périodicité font que seule une partie de la section transversale doit être modélisée. La prise en compte de ces conditions peut, dans la méthode de Trefftz, être considérée en modifiant les fonctions $N_{j}$ dans (4). Si l'on désire étudier les phénomènes existant à l'intérieur d'un disque où la périodicité spatiale est $\theta_{0}$, on peut limiter le domaine d'étude à un secteur angulaire de valeur $\theta_{0}$, à condition d'écrire le potentiel vecteur sous la forme :

$$
A(m)=\sum_{j=1}^{N}\left(\sum_{k=1}^{\frac{2 \pi}{\theta_{0}}} \log \frac{1}{r_{j k}}\right) \beta_{j} .
$$


Les grandeurs $r_{j k}$ représentent les distances du point $m$ à des points $j_{k}$ répartis avec une périodicité $\theta_{0}$ autour du disque (Fig. 3) ; les fonctions $N_{j}$, ainsi définies, assurent automatiquement la périodicité du potentiel vecteur.

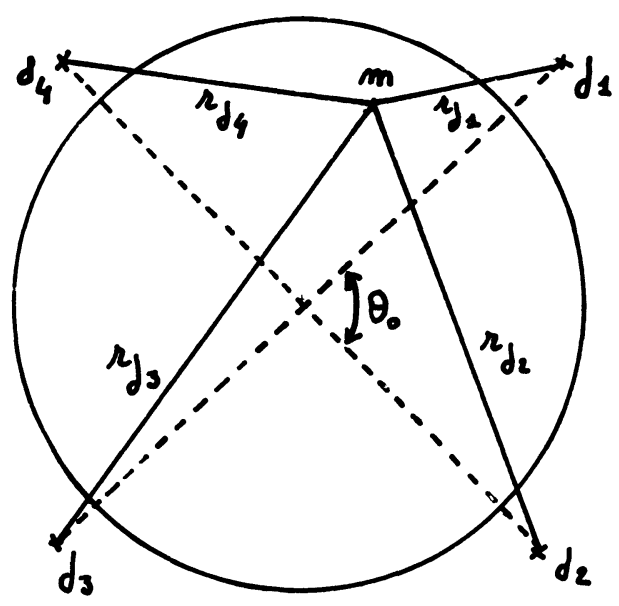

Fig. 3. - Domaine présentant des symétries.

[Domain with symmetries.]

$\mathrm{Vu}$ les conditions aux limites, l'exemple de la figure 4a correspond au cas particulier. où $\theta_{0}=\pi$, seul un demi-disque doit être modélisé. La figure $4 \mathrm{~b}$ représente l'évolution de la dérivée normale de $A$ suivant le choix des fonctions de forme ((4) ou (10)). Les résultats sont excellents si l'on utilise l'expression (10). On s'aperçoit que les fonctions de forme issues de l'expression (4) conduisent à des résultats éronnés, surtout sur les parties latérales du domaine.

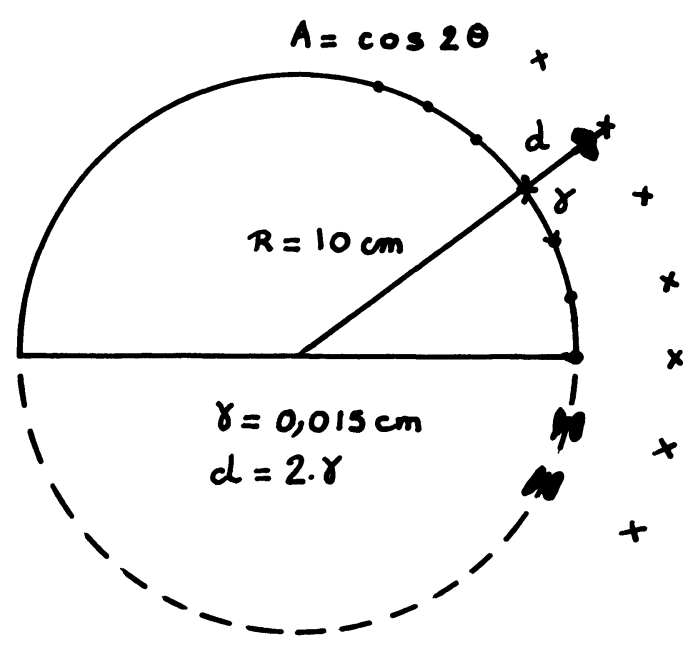

Fig. 4a. - Exemple de domaine modélisé en tenant compte des symétries.

[Studied example with symmetries.]

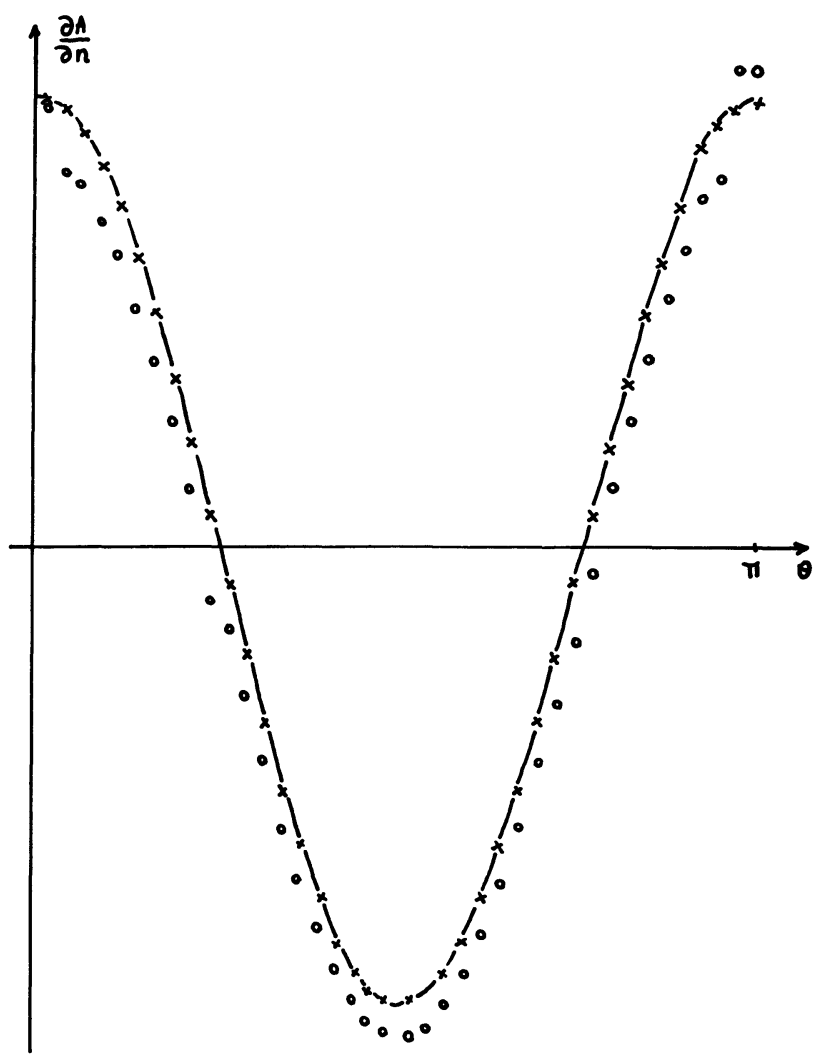

Fig. 4b. - Evolution de la dérivée normale du potentiel vecteur en fonction de $\theta$ avec $\bigcirc \bigcirc \bigcirc A=\sum_{i=1}^{N} \log \frac{1}{r_{i}} \beta_{i}$; $\times \times \times A=\sum_{i=1}^{N}\left(\log \frac{1}{r_{i 1}}+\log \frac{1}{r_{i 2}}\right) \beta_{i} ;-\quad$ solution analytique.

[Normal derivative of vector potentiel as a function of $\boldsymbol{\theta}$.]

\section{Couplage avec la méthode des éléments finis.}

Il peut être intéressant de coupler la méthode des éléments finis et la méthode de Trefftz pour profiter de leurs avantages tout en palliant certains des inconvénients. Ceci nécessite de déterminer la matrice de raideur du domaine modélisé par la méthode de Trefftz.

$\mathrm{Si}$ nous utilisons la méthode de Trefftz pour modéliser un domaine $\Omega$ où l'équation à résoudre est l'équation de Laplace, nous avons alors en tout point $m$ de $\Omega$ :

$$
A(m)=\sum_{j=1}^{N} \log \frac{1}{r_{m j}} \beta_{j}
$$

en utilisant la relation (5), l'expression (11) devient :

$$
A(m)=\sum_{j=1}^{N} \log \frac{1}{r_{m j}} \sum_{l=1}^{N} P_{j l}^{\prime} A_{l}
$$


où $P_{j l}^{\prime}$ est le terme général de la matrice inverse de $P$, ainsi l'égalité (12) est de la forme :

$$
A(m)=\sum_{l=1}^{N} M_{l}(m) A_{l}
$$

avec

$$
M_{l}(m)=\sum_{j=1}^{N} \log \frac{1}{r_{m j}} P_{j l}^{\prime} .
$$

Un domaine modélisé par la méthode de Trefftz apparaît alors comme un élément fini particulier ayant un grand nombre de nœuds et où les fonctions de forme $M_{l}(m)$ vérifient les équations différentiel- les régissant le milieu. La méthode de Trefftz conduit à un macro-élément de forme quelconque qui apparaît comme la généralisation du macroélément uniforme étudié en [5]. La fonctionnelle relative à cet élément sera [3]:

$$
F^{\mathrm{E}}=\frac{\nu_{0}}{2} \iint(\nabla A)^{2} \mathrm{~d} \Omega .
$$

L'équation de minimisation conduit à

$$
\frac{\partial F^{\mathrm{E}}}{\partial A_{i}}=\nu_{0} \iint_{\Omega} \nabla M_{i} \cdot\left(\sum_{k=1}^{N} \nabla M_{k} A_{k}\right) \mathrm{d} \Omega .
$$

En utilisant les propriétés des fonctions vectorielles, l'égalité (15) peut s'écrire :

$$
\frac{\partial F^{\mathrm{E}}}{\partial A_{i}}=\nu_{0}\left[-\iint_{\Omega} \mathrm{M}_{i}\left(\sum_{k=1}^{N} \Delta M_{k} A_{k}\right) \mathrm{d} \Omega+\int_{\Gamma} M_{i} \sum_{k=1}^{N} \frac{\partial M_{k}}{\partial n} A_{k} \mathrm{~d} \gamma\right] .
$$

Etant donné que les fonctions de forme vérifient l'équation de Laplace, nous obtenons :

$$
\frac{\partial F^{\mathrm{E}}}{\partial A_{i}}=\nu_{0} \int_{\Gamma} M_{i} \frac{\partial A}{\partial n} \mathrm{~d} \gamma
$$

La méthode intégrale de Trefftz devant donner dans notre cas une solution compatible avec celle des éléments finis triangulaires du premier ordre, nous supposons $\frac{\partial A}{\partial n}$ constant sur un segment du contour $\Gamma$. Nous calculerons cette valeur à partir de la méthode de Trefftz au milieu d'un segment joignant deux nœuds. Avec les notations de la figure (5), l'expression (17) devient :

$$
\frac{\partial F^{\mathrm{E}}}{\partial A_{i}}=\nu_{0}\left[\left.\frac{\partial A}{\partial n}\right|_{i-1} \int_{\gamma_{i-1}} M_{i} \mathrm{~d} \gamma+\left.\frac{\partial A}{\partial n}\right|_{i} \int_{\gamma_{i}} M_{i} \mathrm{~d} \gamma\right] .
$$

En utilisant la relation (8), (18) s'écrit :

$$
\frac{\partial F^{\mathrm{E}}}{\partial A_{i}}=\sum_{j=1}^{N} S_{i j} A_{j}
$$

avec

$$
S_{i j} \simeq \frac{\nu_{0}}{2}\left[R_{i-1, j} \sqrt{\left(x_{i}-x_{i-1}\right)^{2}+\left(y_{i}-y_{i-1}\right)^{2}}+R_{i, j} \sqrt{\left(x_{i+1}-x\right)^{2}+\left(y_{i+1}-y_{i}\right)^{2}}\right]
$$

où $R_{i j}$ est le terme général de la matrice $[R]$ de la relation (8).

\section{Applications.}

5.1 EXEMPLE NUMÉRIQUE ILLUSTRANT LA VALIDITÉ DU MODĖLE. - Cet exemple est consacré à la résolution de l'équation de Laplace à l'intérieur d'un disque où la condition aux limites sur le contour $\Gamma$ est $A=A_{0} \cos 2 \theta$. La solution analytique d'un tel problème étant connue, nous pouvons tester la validité du modèle. Vu les conditions aux limites, seule la moitié du disque est étudiée. Une partie de celle-ci est modélisée par des éléments finis (Fig. 6a), l'autre est modélisée par une méthode de Trefftz. Les valeurs numériques du potentiel vecteur coïncident avec les valeurs théoriques. Sur le contour $\Gamma_{12}$ (Fig. 6a), l'erreur sur les valeurs maximales du potentiel vecteur est de $1 \%$. La figure $6 \mathrm{~b}$ donne l'allure des équipotentielles vecteurs, elles ne sont représentées qu'à l'intérieur du domaine modé- 


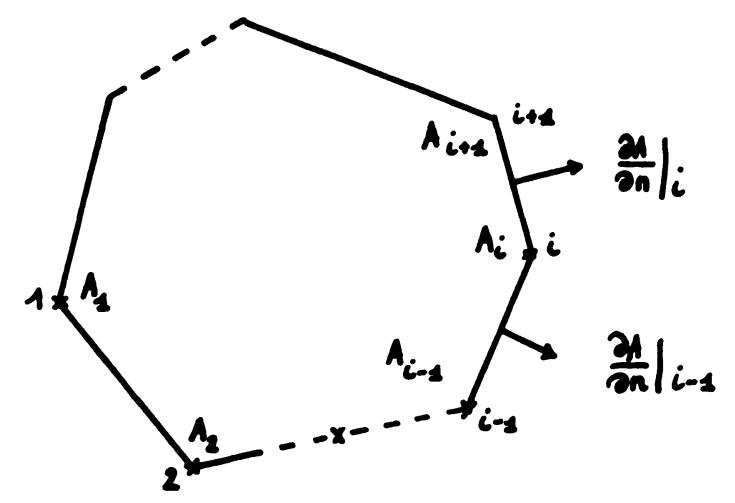

Fig. 5. - Discrétisation du contour $\Gamma$.

[Discretization of the boundary $\Gamma$.].

lisé par des éléments finis. En effet, la modélisation du système peut être considérée comme une modélisation par éléments finis à l'intérieur d'un domaine $\Omega_{2}$ avec une condition aux limites particulière liant $A$ et $\frac{\partial A}{\partial n}$ (voir (8)) sur le contour $\Gamma_{12}$ (Fig. 6a).

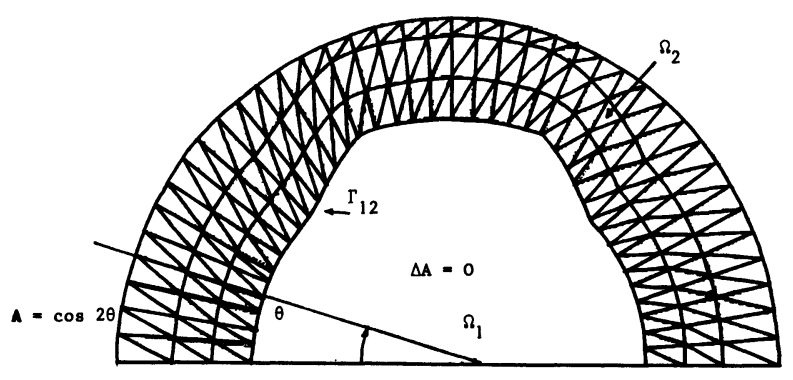

a)

Fig. 6a. - Domaine modélisé par une méthode mixte. [Domain modelled by a hybrid method.]

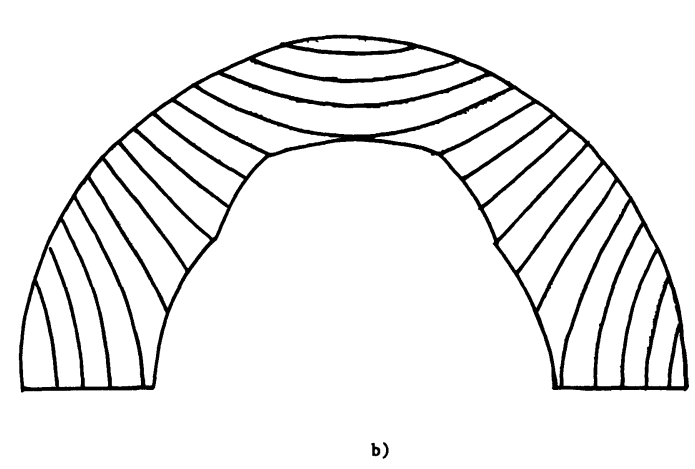

Fig. 6b. - Tracé des équipotentielles vecteurs.

[Flux distribution.]

\subsection{Modélisation d'Un ÉleCtro-Aimant.}

5.2.1 Comparaison avec des résultats expérimentaux. - Nous avons modélisé l'électro-aimant de la figure 7. L'entrefer constitué d'une bande de largeur uniforme est modélisé par la méthode de Trefftz. Le reste du système est représenté par des éléments finis ; chaque quadrilatère du maillage (Fig. 7) est modélisé par deux triangles du $1^{\text {er }}$ ordre. Une telle modélisation pourrait nous permettre de tenir compte de la non-linéarité des matériaux magnétiques. Nous avons calculé, à partir du tenseur de Maxwell, la force d'attraction de l'électro-aimant pour une excitation de 4200 At et pour deux entrefers l'un de 8,32 mm et l'autre de 5,37 mm. Le calcul donne pour le premier, une force de $1477 \mathrm{Nm}$ et pour le second, une force de $2872 \mathrm{Nm}$. Les calculs ont été effectués en supposant les matériaux linéaires ce qui est confirmé par l'expérience. Nous obtenons $8 \%$ d'erreur entre les résultats numériques et expérimentaux [9].

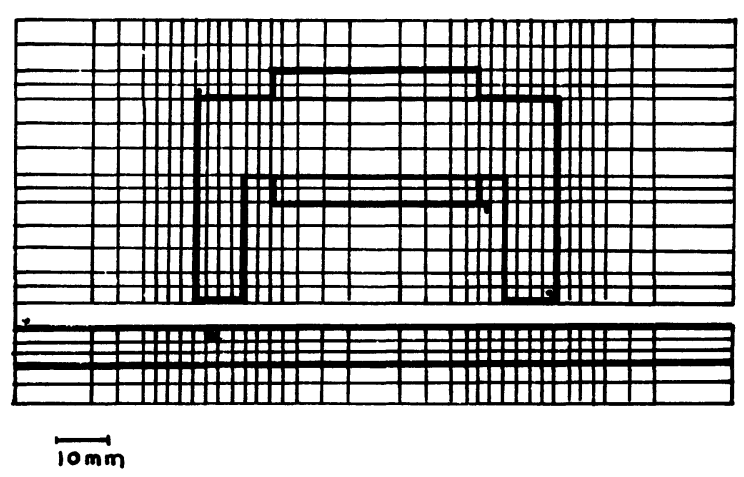

Fig. 7. - Structure de l'électro-aimant.

[Electro-magnet structure.]

5.2.2. Comparaison avec la méthode des éléments finis. - La méthode permettant de modéliser des entrefers non réguliers, nous avons regardé l'influence sur la force d'attraction de 2 dents (Fig. 8) placées sous les cornes polaires de l'électro-

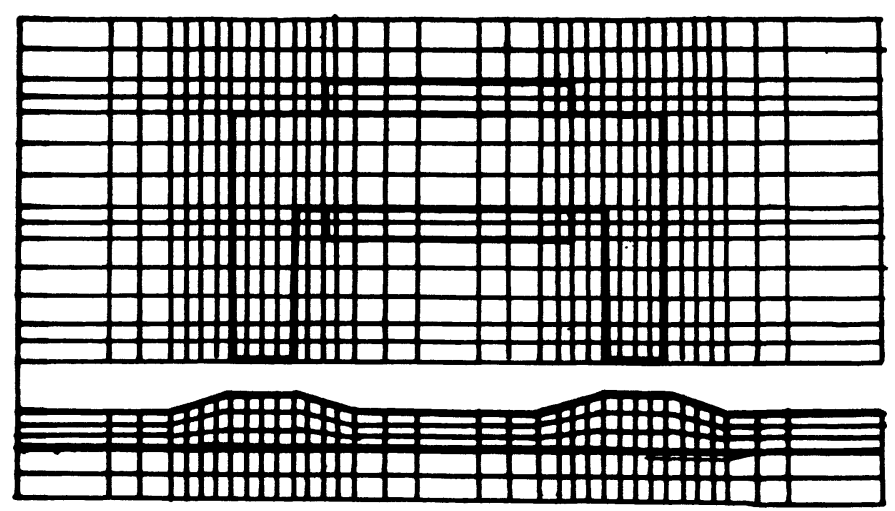

Fig. 8. - Structure de l'électro-aimant avec les dents.

[Electro-magnet structure with teeth.] 


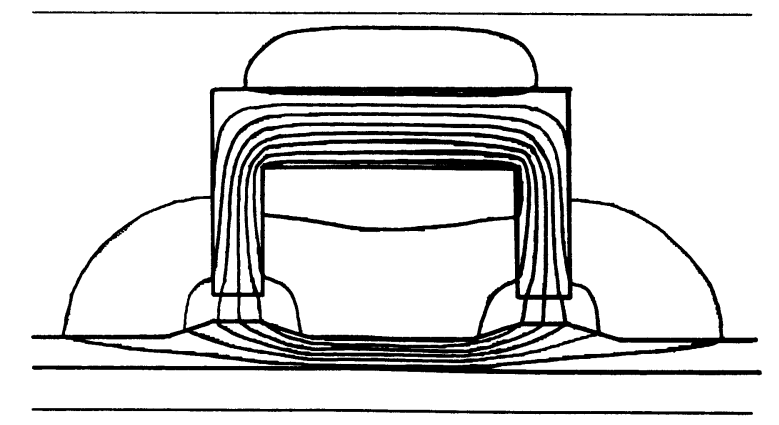

a) $A_{\mathrm{MAX}}=0,01421$

$A_{\mathrm{MIN}}=-0,000682$

Fig. 9. - Tracés des équipotentielles vecteurs pour l'électro-aimant (Fig. 8). a) méthode mixte (E.F. + Trefftz), b) méthode classique par éléments finis.

aimant de la figure 7. Les calculs montrent une diminution de la force de $12 \%$ par rapport à celle obtenue pour un entrefer régulier, de largeur égale à la distance séparant les cornes des dents. Un calcul utilisant uniquement une modélisation par éléments finis confirme ces résultats. Le tracé des lignes de champ obtenu à partir de la formulation mixte est proche de celui obtenu à partir d'une modélisation utilisant uniquement des éléments finis (Fig. 9a, b).

\section{Conclusion.}

Dans cet article, nous présentons une méthode intégrale où la fonction inconnue vérifie rigoureusement l'équation locale gouvernant le problème. Seules les conditions aux limites sont approximées.

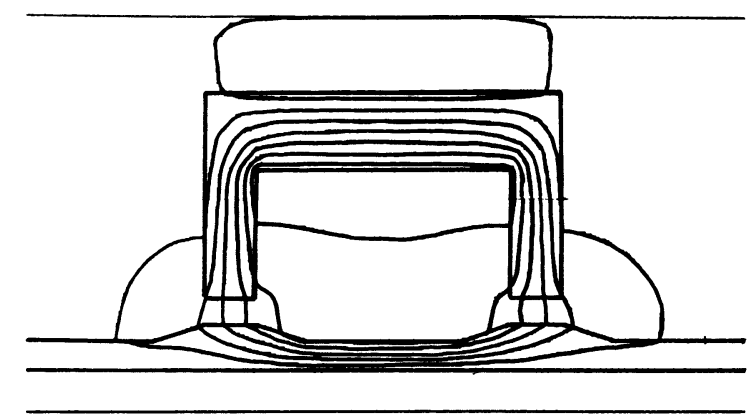

b) $A_{\text {MAX }}=0,01406$

$A_{\mathrm{MIN}}=-0,000669$

[Flux distribution for the electro-magnet (Fig. 8) ; a) hybrid formulation (E.F. + Trefftz) ; b) classical finite elements method.]

Les erreurs d'approximation sont faibles si l'on choisit correctement les points sources et si les fonctions de forme tiennent compte des symétries. Cette méthode a les avantages et les inconvénients d'une méthode intégrale, elle permet de modéliser des domaines infinis mais elle ne peut traiter les phénomènes non linéaires. Dans ce cas, un couplage avec la méthode des éléments finis permet de traiter des problèmes qu'il serait difficile de résoudre en utilisant uniquement des éléments finis. La mise en place informatique de cette méthode par rapport à celle d'une méthode intégrale de frontière est relativement simple. Une contrainte consiste en la définition d'un contour source, ceci semble indiquer que la méthode de Trefftz sera intéressante pour modéliser des domaines de forme relativement simple.

\section{Bibliographie}

[1] Christina, S. and DI NAPOLI, A., Combination of finite and boundary elements for magnetic fields analysis, I.E.E.E. Trans. MAG-19 N $^{\circ} 6$ (1983).

[2] Peng, J. P. and Salon, S. J., Hybrid finite element boundary solutions using half-space Green's functions, J. Appl. Phys. 55 (6) (1984).

[3] Feliachi, M., Razek, A. and Bouillault, F., $A$ synchronous machine dynamic model for magnetic field calculation, Proc. of I.C.E.M., pp. 5153, Budapest, 1982.

[4] Ren, Z., Davat, B. et Lajoie-Mazenc, M., Modélisation des structures magnétiques en tenant compte du mouvement, Actes du colloque Modelec, 22-23 octobre, 1984.

[5] RazeK, A., Coulomb, J. L., Feliachi, M., SabonNADIERE, J. C., Conception of an air-gap element for dynamic analysis of the electromagnetic field in electric machines, I.E.E.E. Trans. MAG-18 (1982) 655-659.
[6] ZienkiewiCZ, O. C., Kelly, D. W. and BetTesS, $\mathrm{P}$., The coupling of finite element method and boundary solution procedures, Int. J. Numer. Meth. Eng. 11 (1977) 335-375.

[7] Patterson, C. and Sheikh, M. A., On the use of fundamental solutions in Trefftz method for potential and elasticity problems (Brebia) 1982.

[8] FeliaChI, M., Contribution au calcul au champ électromagnétique par la méthode des éléments finis en vue de la modélisation numérique des machines électriques, Thèse de docteur ingénieur, LGEP, 1981.

[9] Goby, F., Pascal, J. P. and RazeK, A., Modelling of forces in electromechanical components, Proc. of Electromechanics and Electronics applied to manufacturing process, St. Felice Circeo, Italy, 1985. 